\title{
Research Progress on Phenotypic Plasticity of Invasive Plants in Response to Drought Stress
}

\author{
Zhe Wang $^{1, *}$, Xiaojie Cai ${ }^{1}$, Zhiyi Yin ${ }^{1}$ \\ ${ }^{1}$ School of Life Sciences, Henan University, 475004, Kaifeng, Henan, China
}

\begin{abstract}
Biological invasion seriously threatens ecological stability and social development. One of the bases for forming invasion is alien species' strong phenotypic plasticity. Phenotypic plasticity can help alien species adapt to environmental changes and enhance their invasive ability. China is one of the countries seriously affected by drought. Under the background of environmental drought, exploring the reasons why invasive plants can gain growth advantages and mastering the phenotypic changes of invasive plants in response to drought stress are conducive to helping us understand more about the invasion mechanism of invasive species. In this paper, the research progresses of invasive plants' morphological plasticity and physiological plasticity under drought stress in recent years are reviewed, the invasive plants' morphological and physiological metabolic characteristics under drought are summarized, and the growth advantages of invasive plants are discussed, which provided a scientific theoretical basis for alleviating the serious harm caused by biological invasion to China's natural environment.
\end{abstract}

\section{Introduction}

Biological invasion and habitat loss are the second major causes of global biodiversity reduction, resulting in huge ecological resources and socio-economic losses. Drought stress seriously affects plant growth and crop yield, China is severely affected by drought. During long-term evolution, plants gradually evolved into complex signal networks to adapt to the drought environment by adjusting their morphological characters, physiological and biochemical processes, showing phenotypic plasticity. Phenotypic plasticity exists widely in various organisms; it not only helps maintain the normal growth of plants when the habitat changes but also plays a key role in the successful invasion of alien species. Compared with native species, invasive species have advantages in survival, competition and reproduction [1], while the role of phenotypic plasticity in driving invasive species to produce growth advantage has been widely concerned by researchers. Because invasive plants generally have strong phenotypic plasticity to soil moisture, studying the morphological plasticity and physiological plasticity superiorities of invasive species compared with native species under specific stress such as drought stress is helpful to understand the mechanism of biological invasion, find suitable solutions to solve the problem of biological invasion and promote national economic development.

\section{Biological invasion and phenotypic plasticity}

With the increasing speed of international trade and population movement, more and more creatures have been intentionally or unintentionally introduced into habitats where they had never been before. When these species expand and grow explosively, and become detrimental to the ecological characteristics and social resources of the original ecological system, they are called invasive alien species. Invasive species can change the species composition structure of the community, break the balance of the ecosystem and trigger community structure and ecological pattern to change [1] The biological invasion has become one of the five major global environmental problems in the 21st century, posing a serious threat to the ecological environment, human health and social development [2].

The invasion of alien species in their non-native places is based on their invasive ability and the invisibility of the environment [3], the key of biological invasive ability is phenotypic plasticity. Phenotypic plasticity exists widely, which is reflects in all organisms in nature. The morphological characteristics of most organisms are not only affected by genetic materials but also can vary with environmental factors (such as temperature, humidity, moisture, nutrition, etc.). When the environment changes and stresses organisms' growth, organisms will maintain their normal metabolic development through pursuing advantages and avoiding disadvantages. Plants are usually immobile. Under 
environmental stress, the damage caused by stress is often alleviated through changing plants' morphological characters and physiological and biochemical reaction processes. For example, under drought stress, plant leaves curl to reduce transpiration and water loss, lower their temperature; if the stress continues, in order to grow, plants will produce stable morphological changes, such as in desert areas, plant roots have a larger average connection length, which is convenient for the plant to absorb more water and nutrients [4]. Adaptive growths of plants deeply depend on phenotypic plasticity, which enhances the invasion ability of alien invasive plants.

\section{Damage of drought stress to plants}

Drought has a serious impact on the whole process of plant growth such as seed germination, vegetative growth and reproductive growth [5]. Under drought stress, because seeds are difficulty in absorbing water, which reduces vigor and delays germination. Plant tissue loses water which reduces the activity of reactive oxygen species scavenging enzymes, causes oxidative stress, membrane structures, permeability changes, and in turn lead to cellular inclusion exodus [6][7]. Leaves shrink and curl, light-receiving area decreases, $\mathrm{CO}_{2}$ acquisition is inhibited, which ultimately weaken photosynthesis, restrict plant growth and reduce crop yield. The growths of stems, leaves, roots and fruits of plants are inhibited, and the growth of main roots gradually stagnate, which even leads to the plant death in severe cases.

\section{Morphological plasticity and physio-logical plasticity of invasive plants under drought stress}

According to character types, phenotypic plasticity can be divided into morphological plasticity, physiological plasticity, behavioral plasticity and so on [8]. Usually, plants adapt to drought conditions by adjusting their morphological characteristics, physiological and biochemical processes. Therefore, we will discuss the growth advantages of invasive plants from their morphological and physiological plasticity under drought stress.

\subsection{Morphological plasticity of invasive plants under drought stress}

Under drought stress, Biomass reduction is one of the most obvious effects, the overall morphological characteristic of plants is: aboveground parts' biomass decreasing, leaves wilting, any degree of water deficit will lead to aboveground parts' growth limited; for ensuring plants absorb water, the root system will elongate grow, underground parts' growth get a relative promotion. The photosynthetic products are preferentially distributed to the root system, then the aboveground parts' biomass decreases. So the root-shoot ratio keeps increasing [9]. For example, Solidago canadensis $L$. was prone to reduce the water supply to stems under drought stress, thus allocating more resources to leaves and roots, changing biomass allocation is the embodiment of drought tolerance; Besides, it was able to grow normally in a wide range of soil moisture, representing strong phenotypic plasticity [10]. Under an identical drought condition, the seedlings of invasive Chinese Tallow Tree had higher plant height, larger plant height growth and stem diameter than that in native, meanwhile, there were significant differences between invasive species and native species in terms of crown biomass and total biomass; though there was no significant difference in root biomass, the invasive species possessed higher root biomass and the smaller root-shoot ratio [11], and the plants with better drought tolerance usually have smaller root-shoot ratio than the plants with poor tolerance, which all demonstrated that invasive Chinese Tallow Tree has better drought tolerance. Under continuous drought stress, plant height, leaf number, leaf area, root length, average root diameter and other parameters of Xanthium italicum decreased significantly. However, with the continuous drought stress, there was no obvious difference in related parameters, and Xanthium italicum had no death, indicating it has strong drought resistance, which may be the reason for its successful invasion [12]. To sum up, invasive plants generally show stronger morphological plasticity to soil moisture under drought stress.

\subsection{Physiological plasticity of invasive plants under drought stress}

Plants respond to drought stress through complex signal networks, physiological reactions such as adjustment of photosynthetic pigment content and utilization rate, intracellular osmoregulation and initiation of the antioxidant system all play important roles. Germination parameters such as germination rate, germination index and vigor index can be used to measure the drought resistance of plants [13], and these parameters will gradually decrease with the aggravating of drought. Under drought stress, the chlorophyll utilization rate of plants increased, chlorophyll content and the proportion of each component changed. With the further drought, cells lose water continuously, while plants increase cytosol by increasing osmoregulatory substances to reduce the osmotic potential to maintain cell shape, and enhance the stress resistance. In this process, some organic osmoregulatory substances like proline, betaine, soluble sugars, soluble proteins and some ions in plant cells play important roles [14][15]. In order to preserve plants from oxidative stress, the antioxidant system composed of the enzymatic system and non-enzymatic antioxidant system provides an effective antioxidant mechanism for plants [16]. Invasive plants also have such physiological plasticity regulation, and usually have stronger physiological plasticity to drought stress than native organisms. Under simulated drought conditions by PEG, the germination rate and germination index of the Censhrus Spinfex decreased with the increasing of PEG concentration; however, when PEG reached a higher concentration, some seeds could still germinate, which 
indicated that Cenchrus spinifex has strong drought resistance and physiological plasticity [17]. The chlorophyll content of Conyza canadensis decreased, but the ratio of chlorophyll $\mathrm{a} / \mathrm{b}$ was higher than that of the normal growth control, and the increase of this ratio meant that the light capture of leaves decreased, avoiding the damage caused by photooxidation, showing strong stress resistance [10][18]. With the drought going on, the membrane lipid damage of Erigeron canadensis L. was serious, and the accumulated content of MDA increased gradually. Free proline began to accumulate in large quantities after the moderate drought period, and the content of soluble proteins increased continuously. The soluble protein content under high-intensity stress was more than that under low-intensity stress, which makes the osmotic potential decreased and remained stable; the activity of superoxide dismutase increased during the whole drought process, and the activity of peroxidase kept rising in the moderate drought range [19]. To sum up, under drought stress, invasive plants can maintain normal growth through physiological plasticity and utilize various reaction mechanisms to adapt to diverse habitats and gain the possibility of invasion. In addition, invasive plants usually have stronger restorability, for example, after drought and rewatering, the maximum PSII original light energy conversion efficiency of Wedelia trilobata appeared overcompensation phenomenon, which was higher than that of Wedelia chinensis. The stronger environmental recoverability is also the reason why invasive plants can gain growth advantages [20].

\section{Prospect}

Since the 18th National Congress of the Communist Party of China, the country has vigorously carried out the construction of ecological civilization, although China's ecological environment has been significantly improved on the whole, it is still urgent to alleviate the ecological invasion problem. Phenotypic plasticity is the key to the successful invasion of invasive plants. Further studying on phenotypic plasticity of invasive plants can help us better understand the reasons why invasive plants have advantages in competition with local plants, explore the mechanism of a successful invasion of invasive plants and finally eliminate the impact of biological invasion on ecological balance and national economy.

Successful invasive alien species can pose a threat to the ecological environment, they often gain growth advantages of the invaded place, which can absorb more water and nutrients under the same growth environment, show better stress response under environmental stress. Research by Xinjiang Institute of Ecology and Geography, Chinese Academy of Sciences shows that the situation of drought trend zone in China will be even more terrible in the future, and the drought duration will be longer [21]. Under the background of national water and soil environment changing, the research on the drought stress response of invasive plants is more targeted. Through mastering the reasons why invasive plants can absorb more water and gain growth advantages by changing their phenotypes under drought can help us have a deeper understanding of the invasion mechanism of alien species.

The reasons why invasive plants possess growth advantages in invaded areas and possess stronger water or nutrient use efficiency under the same conditions have not been completely resolved. Sultan thinks that even slight phenotypic variation caused by environmental changes may have biological significance [22], while phenotypic plasticity is only a part of the causes of phenotypic variation, and its relationship with higher phenotypic variability is not clear. Whether invasive plants can adapt to the environment better through stronger and more phenotypic remodeling under environmental stress is still unknown. At present, describing phenotypic plasticity quantitatively based on the qualitative description, and accurately evaluating the role of phenotypic plasticity in the invasion process is necessary.

The comparative study on the responses of invasive plants and native close-up plants under specific stress needs to be further improved, especially in the aspect of root phenotype. The phenotypic plasticity of invasive plants can be grasped more clearly and comprehensively by exploring the root morphology, anatomical structure and other factors that change obviously with the environment. By comprehensively analyzing the differences of morphology, physiological and biochemical processes, the molecular level between invasive plants and native close-up plants is conducive to exploring phenotypic plasticity in-depth, revealing the advantages of invasive plants' water, nutrition and light absorption and utilization, helping us deeply understand the response mechanism of invasive plants, and then solve ecological problems such as serious harm caused by plant invasion to local species.

\section{References}

1. Ye YH et al. (2020) Impact of biological invasion on natural resource assets and its application in natural resource balance sheet preparation. Journal of Ecological Environment, 29 (12): 2465-2472.

2. Meyerson L A, Carlton J T, Simberloff D, et al.(2019) The growing peril of biological invasions. Front Ecol Environ,17(4): 191.

3. Geng YP, Zhang WJ, Li B, Chen JK. (2004) Phenotypic plasticity and invasive ability of exotic plants. Bio-diversity, (04): 447-455.

4. Shan LS. (2013) Studies on the morphological structure and function of roots and drought resistance physiology of typical desert plants in Northwest China. Gansu Agricultural University.

5. Hussain, H.A., Hussain, S., Khaliq, A., et al. (2018) Chilling and drought stresses in crop plants: implications, cross talk, and potential management opportunities. Front Plant Sci, 9: 393.

6. Reddy A R, Chaitanya K V, Vivekanandan M.(2004) Drought-induced responses of photosynthesis and 
antioxidant metabolism in higher plants. Journal of Plant Physiology, 161(11): 1189-1202.

7. Tatar O., Gevrek MN.(2008) Influence of water stress on proline accumulation, lipid peroxidation and water content of wheat. Plant Sci. 7(4): 409-412.

8. Zhai SH, Wang P, Sheng LX.(2017)Phenotypic plasticity of plant functional traits in competition environments. Journal of Beihua University (Natural Science Edition), 18 (4): 538-546.

9. Zheng, M., Tao, Y., Hussain, S., et al.(2016) Seed priming in dry direct-seeded rice: consequences for emergence, seedling growth and associated metabolic events under drought stress. Plant Growth Regul, 78: 167-178.

10. Ge JL, He JQ, Sun XF, Chen Q.(2010) Ecological responses of the invasive alien plant Solidago canadensis to changes of soil water content. Journal of Northwest Botany, 30(03): 575-585.

11. Ge LH.(2020) Effects of drought, salinity and nutritions deficiency on growth and antioxidation of native and invasive Chinese Tallow Tree.Henan University.

12. Hu XY.(2018) Morphology and physiological response of invasive plant Xanthium italicum to drought stress. Shenyang University.

13. Qi SY et al. (2014) Seed germination of invasive species Galinsoga parviflora under drought stress simulated by PEG. Chinese Journal of Ecology, 33 (05): 1190-1194.

14. Wu JS, Zhang JH, Li RJ et al.(2017) The Plant's physiological mechanism and adaptability to drought stress. Journal of Shanxi Agricultural University (Natural Science), 37 (06): 452-456.
15. Wei A., He C., Li B. et al.(2011) The pyramid of transgenes TsVP and BetA effectively enhances the drought tolerance of maize plants. Plant Biotechnol J,9(2): 216-229.

16. Zhao LY, Deng XP, Shan L.(2005) The response mechanism of active Oxygen species removing system to drought Stress. Journal of Northwest Botany, (02): 413-418.

17. Lei Q, Zheng GC, Shi LY, Tian X, Zhou LY.(2016) Effects of $\mathrm{NaCl}$ and PEG stress on seed germination of Cenchrus Pauciflorus. Journal of Inner Mongolia University for Nationalities (Natural Science Edition), 31(06): 492-496.

18. Wang XH.(2015) Invasion mechanism of alien plant- Conyza canadensis L. Shenyang Agricultural University.

19. Zeng ML, Luo Q, Zhang H, Yang D, Ma DW. (2007) The invasive plant Erigeron canadensis $L$. preliminary study on physiological and ecological adaptation. Journal of Sichuan Normal University (Natural Science), (03): 387-390.

20. Song LY et al. (2009) Effects of drought stress and rehydration on chlorophyll fluorescence characteristics in leaves of invasive Wedelia trilobata. Acta Ecologica Sinica, 29(07): 3713-3721.

21. Jinlong Huang et al.(2018) Analysis of future drought characteristics in China using the regional climate model CCLM. Springer Berlin Heidelberg, 50(1):507-525.

22. Sultan,S. E.(1992) What has survived of Darwin's theory? Phenotypic plasticity and the Neo-Darwinian legacy. Evolutionary Trends in Plants, 6: 61-71. 